\title{
An Autoencoder-Based Response Time Model and Its Application in Anomaly Detection
}

\author{
Yiqin Pan \\ University of Wisconsin-Madison \\ Edison M. Choe \\ Graduate Management Admission Council
}

\begin{abstract}
Most psychometric models of response times are primarily theory-driven, meaning they are based on various sets of assumptions about how the data should behave. Although useful in certain contexts, such models are often inadequate for the complexities of realistic testing situations and display a poor fit on empirical data. Therefore, as a functional alternative, the present study proposes a data-driven approach, an autoencoder-based response time model, to modeling response times of correctly answered responses. Also, this study introduces the application of the proposed model in anomaly detection (including aberrant examinee and item detection). The result shows this model has an acceptable performance in both response time modeling and anomaly detection.
\end{abstract}

Key words: Response Time, Autoencoder, Anomaly Detection

\section{Introduction}

Most psychometric models of response times are primarily theory-driven, meaning they are based on various sets of assumptions about how the data should behave (De Boeck, \& Jeon, 2019). However, under most situations the mechanisms control the process of responding are not entirely known and, for a particular test, it is unclear to which degree the mechanism influences the response process. In this way, although theory-driven models are useful in certain contexts, such models are often inadequate for the complexities of realistic testing situations and display poor fit on empirical data.

The latest machine learning techniques are quickly changing the landscape across different industries as the technology becomes more feasible and affordable. Machine learning is a program that learns from data to make predictions or decisions about unknown events without explicit instructions. Machine learning is a highly interdisciplinary field, building on many scientific domains, such as artificial intelligence, statistics, optimization theory, and information theory. Also, it can be applied to a variety of problems, including data mining, 
recognition systems and recommendation engines. Different from statistics, where the goal is making inferences from samples, machine learning algorithms specialize in recognizing generalizable predictive patterns. To be specific, statistical methods concentrate on explicitly verifying assumptions about the problem and refining the specified models, or providing quantitative statements about the confidence for the models; machine learning methods focus on forecasting unseen outcomes while making minimum assumptions about the datagenerating process. Thus, statistical models are chosen based on our domain knowledge in statistics, while machine learning models are chosen relying on their empirical capabilities.

Data modeling, extract of data features at high levels of abstraction, is also a challenging task in machine learning, and numerous algorithms have been proposed to deal with it. Among the commonly used techniques, neural networks are one promising avenue, much research has successfully applied neural networks to model complex data. For example, researchers in speech recognition applied deep neural networks to conduct acoustic modeling and recognize speech (e.g., Hinton, Deng, Yu, Dahl, Mohamed, Jaitly \& Kingsbury, 2012), researchers in computer vision used convolutional neural networks to modeling images and detect objects (e.g., Redmon, Divvala, Girshick \& Farhadi, 2016), and researchers in natural language processing (NLP) use recurrent neural networks to modeling language and serve as the core of other NLP tasks (e.g., Cho, Van Merriënboer, Gulcehre, Bahdanau, Bougares, Schwenk, \& Bengio, 2014). These data (speech, pixel and text) shares similarity with the response time data. These data are all complex data with many not-clearly-known factors which influence the observed simultaneously. For example, when an image is provided to conduct face recognition, even if it is known that the provided image might be influenced by posture and facial expression, the extent to which these factors influence this particular image is unknown, and other factors, like lighting conditions, might be missed if only these two factors are considered. As mentioned above, the same characteristics exist in the response time data. There are many mechanisms (e.g., solution behavior and rapid-guessing behavior) which may influence the response process, however, for a particular test, it is not clearly known to which degree these mechanisms influence the response process, and it is not sure if there exist other mechanisms influencing the response process.

Considering the successful applications of neural networks in modeling complex data in other fields and the similarities between the response time data and these complex data, machine learning may have important and useful applications within the field of educational measurement for modeling response time. Thus, the present study proposed a model based on autoencoder, a type of neural network, to modeling response time. The proposed autoencoder- 
based response time model could be applied to (1) modeling response times, including fit in the provided data and estimating examinee-level and item-level parameters; (2) detecting anomaly, flagging aberrant examinees and items based on the difference between the predicted expected response times and the observed ones. The rest of this paper will proceed with the following sections: (a) introduction of neural networks and autoencoder (b) introduction of the proposed response time model and its application in response time modeling and anomaly detection, (c) description of experiment designs and analysis of the results, and (d) discussion of the implications and limitations of this study, and directions for future exploration.

\section{Neural Network and Autoencoder}

The main concept in neural networks is automating the extraction of representations from the data. These algorithms are greatly motivated by the ideas in artificial intelligence, which has the general goal to imitate the human brain's ability to learn and make decisions, especially for complex problems. A human brain contains billions of neurons that are connected to many other neurons, forming a network. When it receives an external stimulus, the signals from input neurons are abstracted progressively through multiple connections among neurons, finally lead to the output neuron. Neural networks are designed similarly to make the machine think like a human being, mimicking the hierarchical learning approach of the human brain.

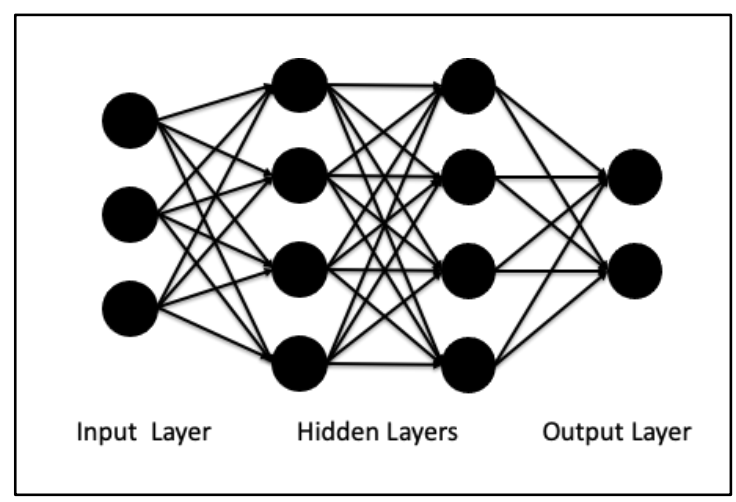

Figure 1 An Example of Neural Network Architecture

Figure 1 shows an example of how researchers implement this idea with neural networks. A neural network is a multi-layer fully connected neural net, which consists of an input layer, multiple hidden layers, and an output layer. Each neuron in the input layer represents a feature; each neuron in the hidden layer is a combination of all neurons in the previous layer, however, its meaning might be uninterpretable by humans; each neuron in the output layer represents a class or category in classification or the prediction value in regression. 
Each edge in the diagram represents a weight. When conducting prediction, given input and weights, the algorithm computes the output by forward propagation. To be specific, each node in the hidden or output layers takes the weighted sum of nodes in the previous layer and passes it through an activation function. The output becomes one of the inputs for the nodes in the next layer. The signal flows from left to right, and the prediction result is in the output layer. The weights associated with all the edges are learned during the training period, and learning the parameters in this deep architecture is a difficult optimization task. One commonly used optimization algorithm is: (1) initialize the weights randomly for all edges; (2) for one training sample, perform a forward propagation to calculate the output and corresponding error; (3) update the weights according to the error. Run steps 2 and 3 repeatedly for each training sample to obtain the trained model.

The functions for acting combinations in hidden layers are called activation functions. Rectified linear unit (ReLU) is the most commonly used activation function in neural networks. Mathematically, it is defined as $f(x)=\max (0, x)$. Compared to other activation functions (e.g., Sigmoid and Tanh), ReLU has a very strong approximation ability, able to approximate most complex functions and produce fast convergence. However, ReLU suffers from a problem named "dying ReLU”. Dying ReLU refers to the situation where ReLU neurons become inactive and only output 0 for any input ( $\mathrm{Lu}$, Shin, Su \& Karniadakis, 2019). If this happens, the "dead" neurons are unlikely to "recover" and become useless in modeling. To solve this problem, some variants of ReLU were proposed. Leaky ReLU is one popular alternative. Instead of zero, leaky ReLU assigns a small slope $(\alpha)$ for negative values. Mathematically, leaky ReLU is defined as

$$
f(x)=\left\{\begin{array}{rl}
\alpha x & \text { for } x<0 \\
x & \text { for } x \geq 0
\end{array} .\right.
$$

In this way, neurons would not have zero-slope part and the "dying ReLU" problem would be solved.

An important characteristic of neural networks is that this model can learn non-linear and complex relationships without explicit instructions. To be specific, the activation functions used in hidden layers are expected to be non-linear activation functions, in other words, the transformations in the deep layers are expected non-linear. In this way, the network can approximate any function, not only linear combination. In contrast, if linear transformations were used, then the input of each layer is a linear function of the output of the previous layer. Under this situation, no matter how many layers of the neural network has, the output is just a linear combination of inputs, and the performance of this model is equivalent to the effect of 
no hidden layer model (which is called perceptron model), whose approximation ability is quite limited. In addition to learning non-linear relationships, different from most machine learning models which are designed based on the understanding of the data or task context, neural networks are designed to learn the features automatically. For example, when a five-layer neural network is used to conduct face recognition, it is reasonable to argue that the model learns edges in the first hidden layer, a more complex feature like eyes in the second layer, facial expression in the third layer, and outputs the recognition result in the output layer. However, the learned features and the learning order are learned automatically in practice, not necessarily to be like this.

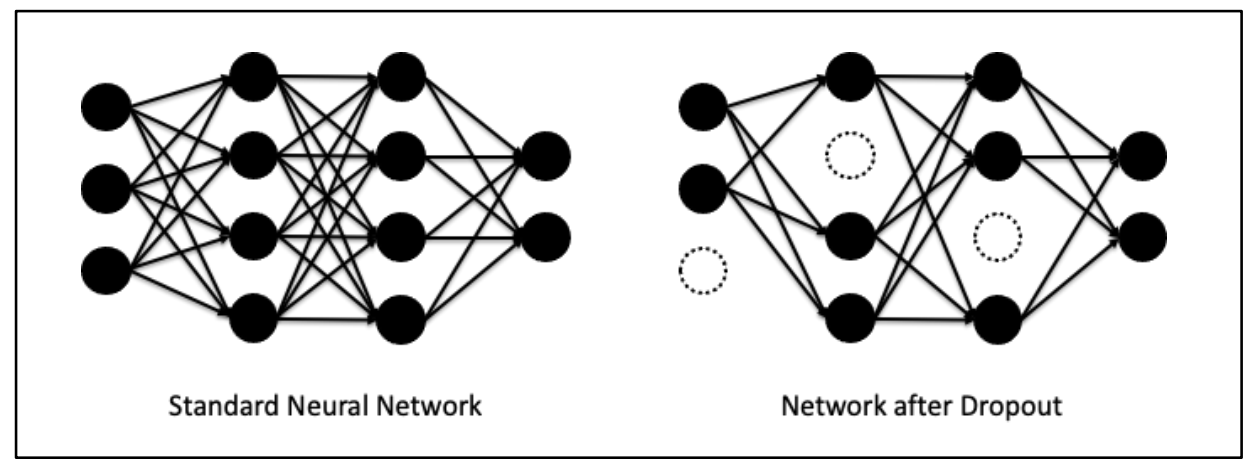

Figure 2 An Example of Dropout Technique

Overfitting is a worth noting problem in learning neural networks. To illustrate, with limited training data, the algorithm may not only learn the real relationship but also the one resulting from noise. Under this situation, the overfitting problem arises. Overfitting prevents the model from perfectly generalizing and many methods have been developed for reducing it. In general, these methods can be categorized into three types: (1) the methods designed for avoiding learning noise, including stopping training early and constraining model complexity; (2) the methods for increasing data size, such as data augmentation; (3) the methods reducing the complexity of the model, including L1 and L2 regularization and dropout. L1 and L2 regularization reduce overfitting by modifying the cost function, while dropout reduces overfitting by modifying the network itself. Dropout strategy randomly drops neurons from the neural network in each training iteration with the number determined by users (see Figure 2). In this way, each iteration trains different neural networks and the models overfit in different ways. As a result, the overall overfitting is reduced. A significant advantage of dropout is that it works well with nearly any model or training procedure, while the trade-off is that the model should be large, and more iterations are needed during the training. 


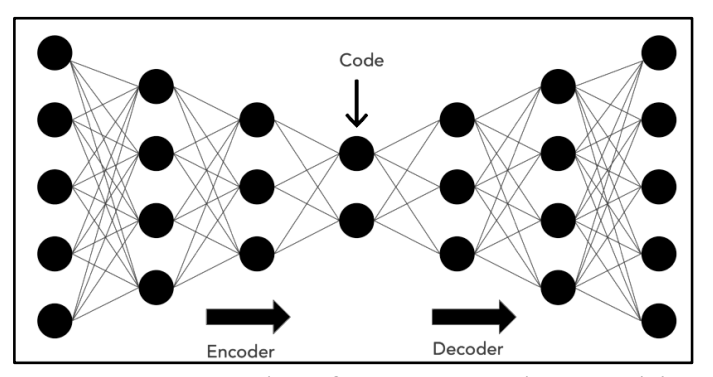

Figure 3 An Example of Autoencoder Architecture

Here we introduce one type of neural network, autoencoder. Autoencoder is a type of neural network which replicates the data from the input to the output in an unsupervised manner. To clarify, a common application of the neural network is predicting a target vector $y$ from an input vector $x$; while autoencoder aims at learning some reduced representations of the input based on which the output layer could be reconstructed. In other words, the autoencoder first conducts dimensionality reduction and then tries to reconstruct the input based on the reduced representations. Thus, the target values are set to be equal to the input and the model is an unsupervised learning algorithm. To implement this idea, an autoencoder is designed to consist of three components (see Figure 3): an encoder, which is a fully connected neural network that compresses the input into a latent space representation; a code that contains the reduced representation of the input; and a decoder, which is a neural network similar to the encoder, responsible for reconstructing the input back from the code. A basic autoencoder learns its parameters by minimizing the reconstruction error and the minimization is usually done by stochastic gradient descent (SGD, Najafabadi, Villanustre, Khoshgoftaar, Seliya, Wald \& Muharemagic, 2015). Same as other neural network models, one important advantage of the autoencoder is that this model can learn both linear and non-linear complex relationships. The present study would propose a response time model based on this type of neural network.

\section{Framework of Autoencoder-Based Response Time Model}

In this section, we introduce (1) the autoencoder-based response time model, (2) how to use the proposed model to modeling response times; (3) how to apply the proposed model to detect aberrant examinees and items.

\section{Autoencoder-Based Response Time Model}

As mentioned above, the response time data is generated through interactions of several known/unknown factors, and for a particular testing, the impact size of each factor is usually not known with certainty. Since autoencoders can automatically learn hierarchical representations and complex relationships in an unsupervised manner, the present study 
proposes a response time model based on the autoencoder model, each part of the model is designed as the following.

Input Layer As the goal of this model is reconstructing response time, each neuron in the input layer represents a response time and the number of neurons is the length of the test. In other words, the input is a response time vector of an examinee to all the items in a test. As normalization can stabilize the learning process and dramatically reduce the required number of training epochs (Santurkar, Tsipras, Ilyas \& Madry, 2018), although neural networks do not impose any restrictions on the input variables (e.g., the distribution of the variables), to make the learning more stable and easier, this approach uses log response times instead of original response times as input (in the following paper, we simply use response time to refer log response time). In addition, as the response times of incorrect responses might follow a different model from the one of the correct responses, this approach is for modeling the response time of correct responses only, the strategy for replacing the times of incorrect answers and completing the times of not-administered items varies by the function of the model (modeling response times or aberrance detection) and is introduced below.

Encoder The number of layers in the encoder and the neuron number of each layer depends on the test length. This approach follows a common strategy for hidden layer structure design: the neuron number of a specific layer is half of the one of its previous layer, with the last layer in the encoder pre-defined. In other words, the neuron number decreases by half until its half (or the ceiling of its half) is not greater than the defined code dimension. For example, if the test length is 30 and the code dimension is 1 , the neuron numbers from the input layer to the code are $30,15,7,3,1$.

Code This layer contains the reduced representations of the response time data, the extracted features. Thus, values in this layer could be regarded as the speed $(s)$ parameters of the corresponding examinee. The dimension of the code or the number of features is defined before learning, the strategy for defining this parameter is detailed below.

Decoder As autoencoder model is symmetric, trying to reconstruct response time, the constructure of the decoder is the reverse of the encoder.

Output Layer As autoencoder model tries to replicate the data from the input to the output, output layer is the same as the input layer.

As mentioned above, autoencoder are usually trained by SGD, thus this model selects SDG as the optimizer. As for activation function, this approach uses leaky ReLU and sets $\alpha$ to be a commonly used value, 0.2 . 


\section{Response Time Modeling}

To apply the proposed model to modeling response time, the following components in the proposed model are further designed. First, as the response times of incorrectly answered or not-administered items should be replaced or completed in advance to obtain input or training data, for response time modeling, all of them are set to be 0 . With value 0 , the impacts of these times on the statuses of all the neurons are minimized. Second, the structure of the network is not fixed in the model design, depending on the defined code dimension. In the application, the dimension of the code is initialized to be 1 . However, not all the time the response time data could be reduced into one abstract feature. Thus, if the model fitness does not meet a pre-defined model fitness criterion, instead of stopping training, this approach doubles the dimension of the (e.g., increasing the code dimension from 1 to $1 \times 2=2$ ) and continue training for a new model until the fitness criterion is met. Note that, as the model becomes useless if the number of the feature exceeds the test length, even though the criterion is not met, this approach stops training if the double of the current feature number exceeds the test length, the latest trained model is used as the finalized model. Here, the fitness is evaluated by the coefficient of determination $r^{2}$, calculated using the observed times and the predicted ones with the times of incorrect answered and not-administered items ignored; the predicted times are obtained by inputting the training data into the trained model, then the output is the predicted expected response. Third, since the target is modeling the response time of correctly answered items, the loss function is set to be the mean squared error between input and output response times of correct responses, rather than the input and output vector. Fourth, to avoid overfitting, dropout layers are added after each layer except the code layer and the output layer.

The process of data fitting is the process of model training. After the model is trained by the target dataset, the weights on the autoencoder are the estimated item parameters, describing the response time properties of the items; the reduced representation of the input vector in the code layer is the estimated examinee parameters for the corresponding examinee. To be specific, to estimate examinee parameters for a specific examinee, for this examinee's observed response time vector, this approach first replaces the response times of incorrectly answered and not-administered items by 0 , and then inputs this updated response time vector into the model, the vector output from the code layer is the examinee parameter. Note that, as the values in the code layer are influenced by the scale and signs of the weights, the examineelevel and item-level parameter outputs from one test are comparable but the ones from different tests are not comparable. 


\section{Anomaly Detection}

The application of the proposed model to anomaly detection is based on the difference between the observed response times and the predicted expected response times output from a trained model. The process of anomaly detection is shown in Figure 4.

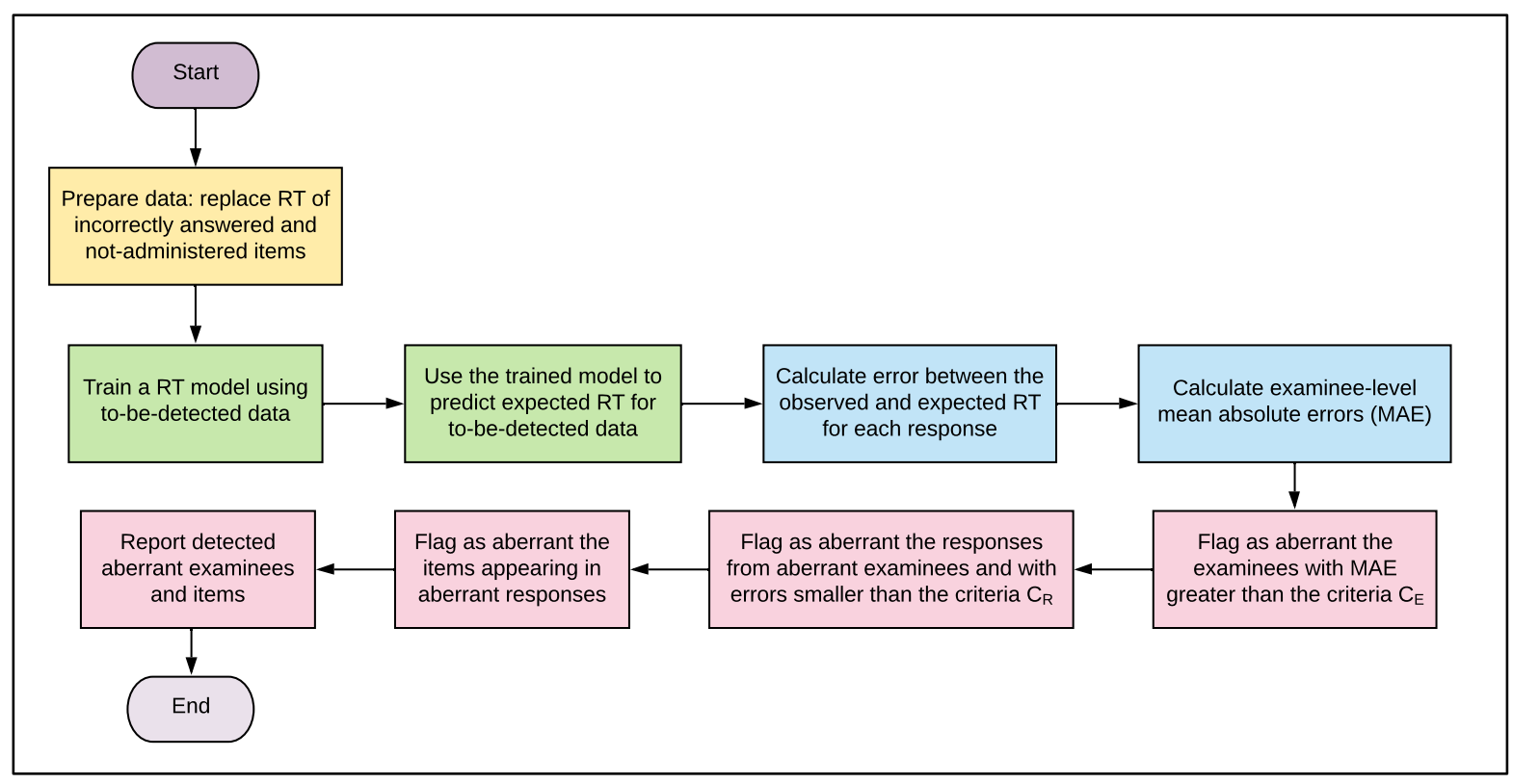

Figure 4 Process of Anomaly Detection

With the goal of detection, the following components in the proposed model are further designed. This approach replaces the response times of incorrectly answered and notadministered items by corresponding examinee's average response time across correct responses; the dimension of the code is fixed to be 1 ; the loss function is set to be a commonly used index, the mean squared error between the input and output vector. The reason why the replacement strategy in anomaly detection is different from the one used in response time modeling is that their goals are different. The purpose of the replacement strategy in response time modeling is to reduce the possible errors from the incorrect answers or not-administered items, setting the corresponding times to 0 might control this kind of error; however, the strategy used here is expected to help make aberrance signal more significant, setting the corresponding times to examinee's average response time increases the number of normal responses and might amplify the aberrance signal. Specifically, since the loss function is the error between the input and out vector, to optimize the model, the model with the replacingby-average strategy would be trained to predict times closer to average compared to the one with the replacing-by-zero strategy. In this way, the impact of aberrance would be weakened, and the aberrance signal could be amplified. 
After the model is trained by the target to-be-detected data, this approach inputs all the target data to the model. The data output from the model is considered as the predicted expected response times, and the difference between observed and expected response times for correct responses is used for detection. This approach first conducts aberrant examinees detection. For a particular examinee, if there is no aberrance existing in the responses, the expected responses should be similar to the observed ones; however, if the responses contain great aberrance, the expected ones would not match the observed ones. In this way, this approach uses the mean absolute error across all correctly answered items to evaluate the aberrance degree of each examinee. If the aberrance degree is greater than the criterion, the corresponding examinee is flagged as aberrant. Here, the criterion is set according to the distribution of examinee-level mean absolute errors, defined as the value which is $C_{E}$ standard deviation units greater than the center (center $+C_{E} \times S D$ ). In the proposed approach, the center is calculated by mode and the standard deviation is calculated using a subset of data. Specifically, when there is no aberrance existing in the data, the aberrance degrees are expected to be distributed normally or symmetrically, however, for the data with aberrance, the right tail of the distribution tends to be longer than expected. Under this situation, we argue the mode of the aberrance degrees would be a better estimation of the center than the mean, and use mode as the center. As for variance, we argue that the degree of dispersion among the data with aberrance degree smaller than the mode would be a more reliable value than the one among all the data, thus use as the variance the average Euclidean distance between the mode and the aberrance degrees which are smaller than the mode. The distance criterion $C_{E}$ is determined according to the distribution of the mean squared errors by the users.

After aberrant examinee detection, the algorithm moves to aberrant item detection. This approach cannot simply apply the strategy for aberrant examinee detection to aberrant item detection. As the model input is the response times of an examinee, the distance between the expected and the observed can reflect the aberrance degree of this particular examinee. However, for a specific item, the great difference between the expected and the observed may be caused by the aberrance of other items rather than this item itself. That is, the model treats the input vector as a whole and conduct prediction not only based on the response time of the corresponding item but also the ones of other items. Under this situation, if the input vector is influenced by the aberrance, not only the aberrant items but also other items will be with expected times greatly different from the observed. Thus, this approach cannot use item-level mean absolute error to detect aberrant items. 
Although the item-level mean absolute error cannot help detecting aberrant items, we argue that the time errors of the correct responses may help. As mentioned above, many normal responses may manifest great difference between their expected and observed response times, however, the responses with very great difference are still most likely aberrant. In this way, it is possible to flag a subset of the aberrant responses and identify aberrant items based on these flagged responses. According to this idea, this approach first uses the errors of correct responses to flag a set of responses, considers as aberrant the flagged responses from aberrant examinees, and then considers as aberrant the items appearing in the aberrant responses. Note that the response flagging is based on the error (the observed-the predicted) distribution rather than absolute error, hence the flagging criterion is set to be $C_{R}$ standard deviation units less than the mode (mode $-C_{R} \times S D$ ), and the variance is the average Euclidean distance between the mode and the errors which are greater than the mode. Same as aberrant examinee detection, $C_{R}$ is determined according to the distribution of the errors by the users.

\section{Experiment 1 Response Time Modeling}

\section{Experiment Design}

As the input of the proposed response time model is the response time vector of an examinee, if this model has a good estimation of the item parameters, the model is expected to successfully conduct reconstruction for the response time vector of a new examinee. In addition, as mentioned above, the examinee parameter output from the code layer can be considered as speed, if this model has a good estimation of the examinee parameters, the output speed is expected to be consistent with the speed parameter for generating the response time data. Based on these ideas, to evaluate the performance of this approach in response time modeling, the present study (1) simulates response data; (2) randomly selects response data of $60 \%$ examinees to train the model; (3) evaluates the performance of model fitting by the fitness of the training data, the performance of item parameter estimation by the model fitness of the remained $40 \%$ data, the performance of examinee parameter estimation by the correlation strength between the estimated speeds and the speed parameters for generating the data.

The present study simulates the response data according to the CAT framework, manipulating two design factors: number of items $(50,100)$ and item pool size $(300,500)$. The number of examinees is fixed to be 500 . Two factors were fully crossed, resulting in $4(2 \times 2)$ study conditions. For each condition, 30 replications were simulated. 
As introduced above, the model fitness criterion is a user-defined parameter to be input to the algorithm, in all the replications, the present study set the criterion to be 0.5 , so as to make the model explain at least $50 \%$ variabilities in the data. As the optimizer is SGD, whose performance sometimes is not very stable, for each particular model structure, this experiment gives the algorithm two additional opportunities to re-train the model if the fitness criterion is not met. In addition, the drop rate for dropout technique was set to be 0.1 ; to ensure the model is fully trained, the learning rate was set to be 0.05 , the batch size was 32 and epoch was 500 .

All programming was done in Python 3.7, the source code is included as supplemental material.

\section{Response Simulation Model}

To generate responses and response times, this study uses a joint IRT and response time model within a hierarchical framework (H-IRTRT; van der Linden, 2007). The first level consisted of the 3PL and lognormal models, and the second level specified the covariance structure between the person parameters and among the item parameters.

In the first level, the 3PL model for generating item responses is:

$$
P\left(x_{i j}=1 \mid \theta_{j}, a_{i}, b_{i}, c_{i}\right)=c_{i}+\frac{1-c_{i}}{1+e^{-a_{i}\left(\theta_{j}-b_{i}\right)}}
$$

where $\theta_{j}$ is the ability of examinee $j, a_{i}, b_{i}$ and $c_{i}$ are the discrimination parameter, difficulty parameter and guessing parameter of item $i$, respectively, and $P\left(x_{i j}=1\right)$ is the probability of examinee $j$ giving a correct response to item $i$. The log response time was randomly generated from

$$
r t_{i j} \sim N\left(\phi_{i}-\zeta_{j}, 1 / \lambda_{i}\right)
$$

where $\zeta_{j}$ denotes the speed parameter of examinee $j, \phi_{i}$ and $\lambda_{i}$ are the discrimination parameter and time-intensity parameter of item $i$, respectively, $r t_{i j}$ is the log response time of examinee $j$ on item $i$.

In the second level, person parameters are defined as following a bivariate normal distribution as below.

$$
\left(\theta_{j}, \zeta_{j}\right) \sim N_{2}\left(\mu_{P}, \Sigma_{P}\right)
$$

with

$$
\begin{gathered}
\mu_{P}=\left(\mu_{\theta}, \mu_{\zeta}\right), \\
\Sigma_{P}=\left(\begin{array}{cc}
\sigma_{\theta}^{2} & \sigma_{\theta, \zeta} \\
\sigma_{\zeta, \theta} & \sigma_{\zeta}^{2}
\end{array}\right) .
\end{gathered}
$$

Following settings were used: 


$$
\begin{gathered}
\mu_{P}=(0,0), \\
\Sigma_{P}=\left(\begin{array}{cc}
1 & 0.3 \\
0.3 & 1
\end{array}\right) .
\end{gathered}
$$

For item parameters, instead of simulating using covariance structures, they present study used the item parameters applied in Choe, Zhang \& Chang (2018). These parameters were also calibrated under the two-level hierarchical framework (van der Linden, 2007), based on real data from a high-stakes, large-scale standardized CAT, which consisted of raw responses and response times from about 2000 examinees with an item pool of 620 items. As the item pool size is fixed to be 500, parameters of 500 items are selected randomly from the item parameter data in each replication.

\section{CAT Simulation Algorithm}

The CAT system was built upon the a-stratification with b-blocking item selection algorithm (Chang, Qian, \& Ying, 2001). Before using the CAT framework to simulate responses, we (1) prepare item and examinee parameters according to the methods mentioned above, (2) stratify items by $b$-parameter values, and then groups them by $a$-parameter values from each $b$-parameter stratum to construct several item strata, in the present study, the number of item strata is fixed to be 5 , as the item pool size is fixed to be 500 , the item pool will be divided into 5 strata of 100 items each, (3) specify EWP and CI by random selection with the number of such examinees/items determined by the particular condition. After these, we iterated following three steps for each examinee:

Estimation of ability: estimate the latest ability $\hat{\theta}$. At the beginning of the test, there is no response data for ability estimation, we initial latest ability by drawing a random value from normal distribution $N(0,1)$. After response data is collect from one or more items, $\hat{\theta}$ is updated by expected a posteriori (EAP).

Selection of item: select the next item given the latest interim $\hat{\theta}$. Suppose $n_{\text {adm }}$ items have been administered to the current examinee and $n_{\mathrm{s}}$ items will be administered among each stratum (as the test length and the number of item strata are fixed to be 50 and $5, n_{s}$ is 10 in the present study), this approach selects as the next item the item with $b$-parameter closet to $\hat{\theta}$ in $\left(\left\lfloor n_{\mathrm{adm}} / n_{\mathrm{s}}\right\rfloor+1\right)^{\text {th }}$ item stratum.

Generation of responses: simulate the response using the H-IRTRT model introduced above.

\section{Experiment Result}

To evaluate the performance of the proposed approach in model fitting, the present study first inputs the training data to the trained model to obtain estimated times, and then 
calculates the following indexes for the estimated and observed response times: coefficient of determination $r^{2}$ (in fact this index is already calculated during training to finalized to autoencoder construction), mean absolute error for response times in log scale $\left(\mathrm{MAE}_{\log }\right)$, rooted mean squared error for response times in log scale $\left(\mathrm{RMSE}_{\log }\right)$, mean absolute error for response times in log scale $\left(\mathrm{MAE}_{\mathrm{org}}\right)$, rooted mean squared error for response times in log scale $\left(\mathrm{RMSE}_{\mathrm{org}}\right)$. Note that, as modeling response times of correct responses is the initial motivation, all the indexes are calculated for correct responses only, the response times of the incorrect responses are ignored. The results are presented in Table 1. In general, this approach has a good performance in model fitting. This model predicts about $67 \%$ of the variabilities in the response times. For the log response times, the MAE is controlled to be about 0.55 and the RMSE is about 0.72 ; in the original scale, the MAE is controlled to be about 1.45 and the RMSE is about 4.8. In addition, the dimension of the code layer is finalized to be 1 in all the replications.

Table 1 Model Fitting Performance

\begin{tabular}{ccccccc}
\hline $\begin{array}{c}\text { Item Pool } \\
\text { Size }\end{array}$ & $\begin{array}{c}\text { Number of } \\
\text { Items }\end{array}$ & $r^{2}$ & MAE $_{\text {log }}$ & MAE $_{\text {org }}$ & RMSE $_{\text {log }}$ & RMSE $_{\text {org }}$ \\
\hline 300 & 50 & 0.686 & 0.545 & 1.447 & 0.714 & 4.836 \\
300 & 100 & 0.657 & 0.564 & 1.503 & 0.743 & 4.600 \\
500 & 50 & 0.692 & 0.538 & 1.430 & 0.706 & 4.917 \\
500 & 100 & 0.679 & 0.551 & 1.431 & 0.726 & 4.822 \\
\hline
\end{tabular}

An index $\left|r_{s, \zeta}\right|$ is used to evaluate the performance of the proposed approach in examinee parameter estimation, $\left|r_{s, \zeta}\right|$ is an absolute value of the correlation coefficient between the estimated speeds $s$ output from the code layer and the speeds $\zeta$ used in the simulation. Here, all the data (not only $60 \%$ training data or the remained $40 \%$ ) are used to conduct evaluation and the estimated speeds $s$ are obtained by inputting all the data to the trained model and outputting the vector in the code layer. The reason why correlation is used to evaluate the performance rather than errors (the difference between the estimated speed $s$ and the speed $\zeta$ used in the simulation) is that, although both $s$ and $\zeta$ are speed parameters, they are estimated from different models, thus there is no point to evaluate the estimation performance by accuracy. Since $\zeta$ is the parameter used to simulate data, if this approach has a good performance in speed estimation, the relative locations of the examinees in speed distribution are expected to be consistent with the ones in simulation. Hence, the correlation is used to compute the evaluation index. Also, as the values in the speed vector are influenced by the scale and signs of the weights, to get rid of the influence of the sign, the absolute value of 
the correlation rather than the correlation coefficient itself is used as the index. Note that, if there is more than one neuron in the code layer, the averages of the estimated speeds are used to calculate the correlation. Table 1 shows the average absolute correlation values among 30 replications. In general, the strength of the correlation is about 0.87 , indicating that this approach has a good performance in speed estimation, or to be more exact, a good performance in describing the relative speed locations of the examinees.

Table 2 Examinee Parameter Estimation Performance

\begin{tabular}{ccccc}
\hline Item Pool Size & \multicolumn{3}{c}{300} & \multicolumn{3}{c}{500} \\
\cline { 2 - 5 } Number of Items & 50 & 100 & 50 & 100 \\
\hline$\left|r_{s, \zeta}\right|$ & 0.869 & 0.861 & 0.874 & 0.873 \\
\hline
\end{tabular}

As the item parameters are the weights on the encoders, it hard to compare these values with the parameters used in the simulation. If this model has a good estimation of the item parameters, the model is expected to successfully conduct reconstruction for the response time vector of a new examinee. Hence, this study evaluates the performance of the proposed approach in item parameter estimation by evaluating the model fit in the testing data (the remained $40 \%$ data). Specifically, this approach first inputs the testing data to the trained model to obtain estimated response times, and then calculates the indexes used in the model fitting evaluation. The results are presented in Table 3. In general, this result is similar to the model fit for training data (see Table 1), showing a good fitness, hence this approach has an acceptable performance in item parameter estimation.

Table 3 Item Parameter Estimation Performance

\begin{tabular}{ccccccc}
\hline $\begin{array}{c}\text { Item Pool } \\
\text { Size }\end{array}$ & $\begin{array}{c}\text { Number of } \\
\text { Items }\end{array}$ & $r^{2}$ & MAE $_{\text {log }}$ & MAE $_{\text {org }}$ & RMSE $_{\text {log }}$ & RMSE $_{\text {org }}$ \\
\hline 300 & 50 & 0.613 & 0.601 & 1.633 & 0.790 & 5.111 \\
300 & 100 & 0.611 & 0.599 & 1.532 & 0.788 & 4.610 \\
500 & 50 & 0.581 & 0.627 & 1.618 & 0.828 & 5.240 \\
500 & 100 & 0.617 & 0.603 & 1.598 & 0.794 & 5.554 \\
\hline
\end{tabular}

For both speed estimation and expected response time prediction, the number of items and the item pool size do not have a significant influence on the performance.

\section{Experiment 2 Anomaly Detection}

\section{Experiment Design}

To evaluate the performance of this response time model in anomaly detection, the present study (1) simulates response data which includes aberrant responses; (2) applies the 
proposed approach to detect aberrant examinees and items, and (3) analyses the performance of this approach under various conditions.

Experiment 2 manipulates two design factors: proportion of aberrant items $(0.1,0.2$, $0.4,0.6)$ and proportion of aberrant examinees $(0.1,0.2,0.4,0.6)$. The test length, the item pool size and the number of examinees are fixed to be 50,500 and 500. Two factors were fully crossed, resulting in $16(4 \times 4)$ study conditions. In addition, baseline condition in which no preknowledge was simulated was also considered, resulting in an additional condition. For each condition, 30 replications were simulated.

The data generating process in this experiment is similar to the one in Experiment 1. The only difference is that this experiment generates aberrant responses. To be specific, before generation, the experiment selects items and examinees as aberrant randomly with numbers determined by the simulation condition. During generation, the method for generating normal responses is the same as the one in Experiment 1. While for the responses of aberrant examinees to aberrant items, the item responses are generated by drawing values randomly from a Bernoulli trial with $p=0.9$ (Eckerly, 2017; Sinharay, 2017), irrespective of the item's difficulty or the examinee's ability; the log response times are obtained by drawing values randomly from a normal distribution, $N\left(-2,1 / 3.5^{2}\right)$ (Choe, Zhang \& Chang, 2018), irrespective of the examinee's speed. In this way, the aberrant responses could be with an expected accuracy of $90 \%$, and their response times could be objectively fast.

As introduced above, the criteria for flagging examinees and responses, $C_{E}$ and $C_{R}$ are user-defined parameters for detection, in all the replications, the present study used 3 as $C_{E}$ and $C_{R}$, a commonly used criterion for flagging outlier in the unit of standard deviation. In addition, same as Experiment 1, this experiment set the learning rate to be 0.05 , the batch size to be 32 and the epoch to be 500 .

\section{Experiment Result}

The present research evaluates the performance of the proposed response time model in anomaly detection from four aspects: false negative and false positive rates for aberrant items and examinees detection. False negative refers to the situation where item $j$ or examinee $i$ is aberrant but not detected; hence the false negative rate for item is computed as the ratio of the number of truly aberrant item which were not detected to the total number of truly aberrant items, the false negative rate for examinee is the ratio of the number of aberrant examinees which were not detected to the total number of aberrant examinees. On the other hand, false 
positive refers to the situation where item $j$ or examinee $i$ is not aberrant but was detected as being one.

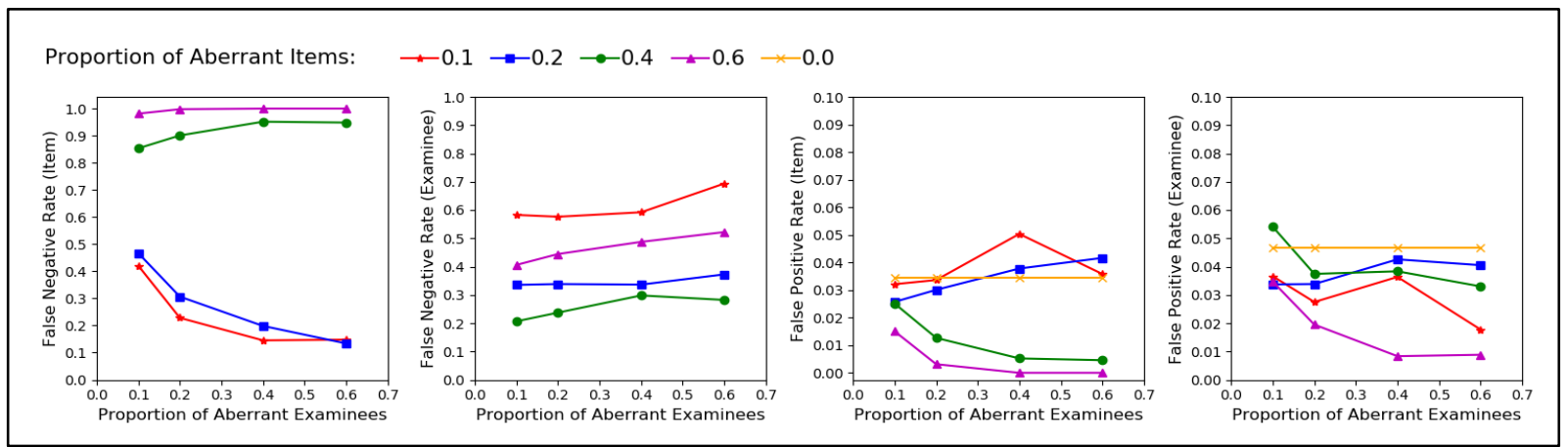

Figure 5 Anomaly Detection Performance

Figure 5 shows the performance of the proposed model in anomaly detection under all conditions. In general, this approach has an acceptable performance in aberrant item detection. Provided the proportion of aberrant items is lower than 0.4 , this approach controls the false negative rates below 0.5 and the false positive rates below 0.05 . In addition, the false negative rates tend to decrease as the proportion of aberrant examinees increases, when the aberrant examinee proportion is relatively high (e.g., 0.6), this approach produces false negative rates around 0.2. However, when the aberrant item proportion is relatively high (e.g., 0.4), the false negative rates become extremely high. As for aberrant examinee detection, this approach also generates acceptable performance. Under most conditions, this approach produces false negative rates lower than 0.5 and controls the false positive rates below 0.06 . The false negative rates are relatively low provided the aberrant item proportion is moderate, while the aberrant examinee proportion does not show a significant impact on the error rates.

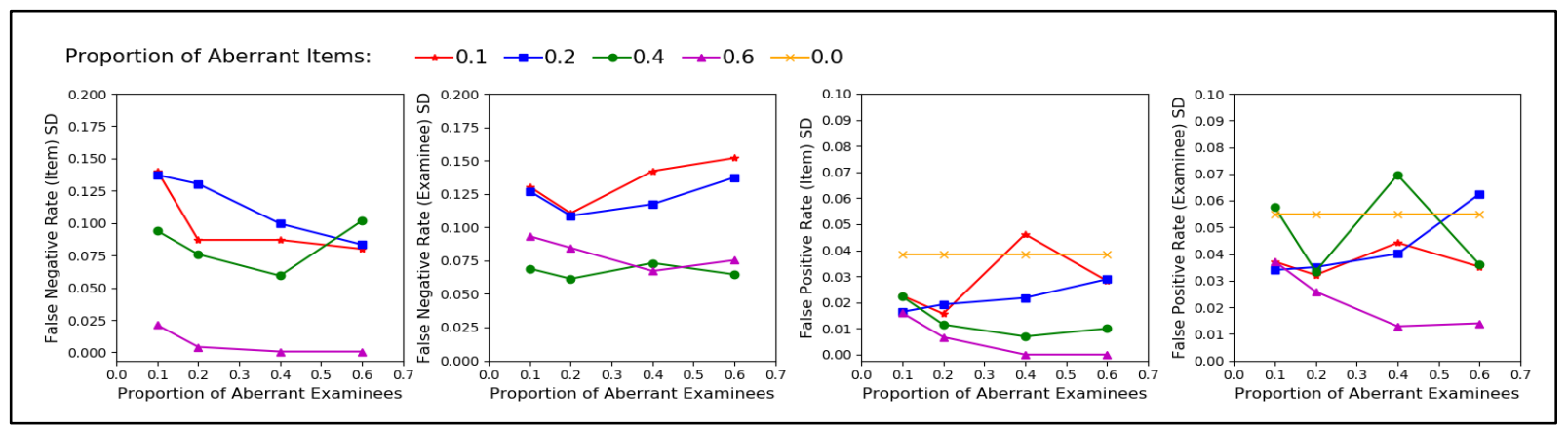

Figure 6 Standard Deviations of Anomaly Detection Performance

Figure 6 shows the standard deviations of the error rates across 30 replications. The standard deviations of false negative rates are below 0.15 and the ones of false positive rates are below 0.07. In general, this approach has stable performance across replications. 


\section{Real Data Experiment}

The dataset from a high-stakes graduate business school admission test was used to demonstrate the performance of the autoencoder-based response time model in response time modeling. This dataset contains three separate timed subsections, linguistic skills (LS), logical reasoning (LR), and quantitative skills (QS). Each subsection is linear-on-the-fly (LOFT) testing. The item pool sizes, the number of items administered, correct rates are presented in Table 4; the log response time distribution of correctly answered items are presented in Figure 7.

Table 4 Item Parameter Estimation Performance

\begin{tabular}{cccc}
\hline Subsections & Item pool size & Administered item size & correct rates \\
\hline LS & 160 & 32 & 0.49 \\
LR & 200 & 40 & 0.44 \\
QS & 240 & 48 & 0.38 \\
\hline
\end{tabular}

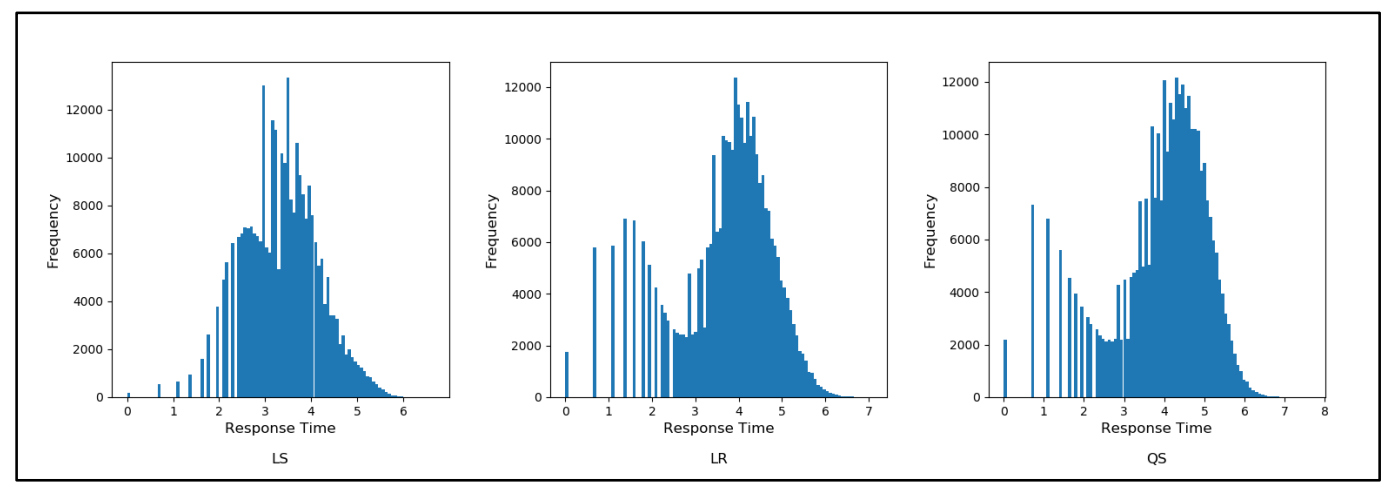

Figure 7 Response Time Distribution of Correctly Answered Items

The proposed model was first applied to modeling response times in the empirical data.

For each subsection, the present study (1) randomly selects response data of $60 \%$ examinees to train the model; (2) evaluates the performance of model fitting by the fitness of the training data; (3) evaluates the performance of item parameter estimation by the model fitness of the remained $40 \%$ data; (4) plots the estimated examinee parameters.

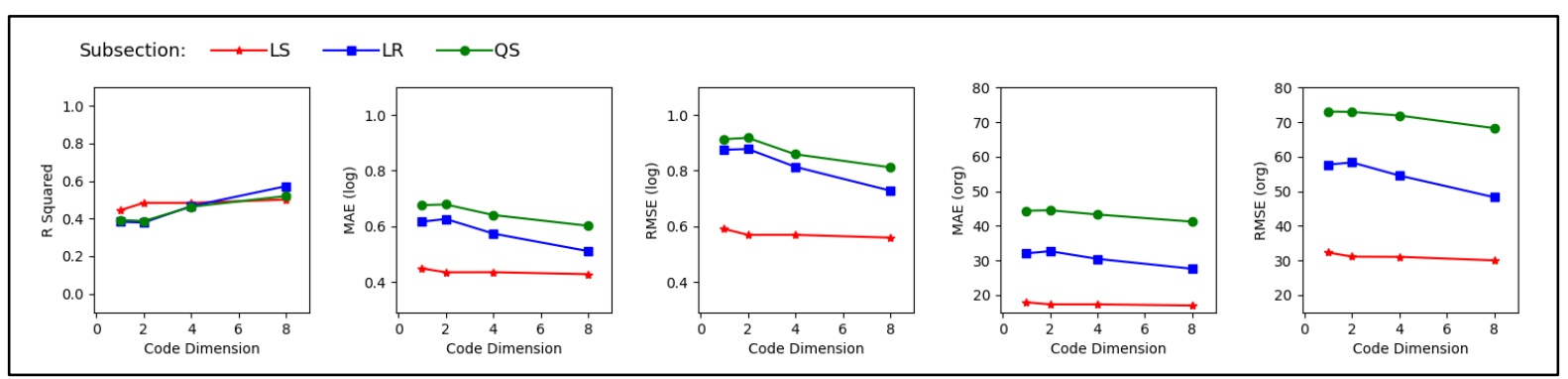

Figure 8 Model Fitting Performance in Empirical Data 
For model training, all the parameters and hyper-parameters are set to be consistent with the ones in Experiment 1: the model fitness criterion was 0.5, the dropping rate was 0.1, the learning rate was 0.05 , the batch size was 32 and the epoch was 500 . Figure 8 shows the model fitness for three subsections across different code layer neuron numbers. In general, this approach performs well in response time modeling even when the code layer neuron number is 1 . For all the subsections, the method produces $r^{2}$ higher than 0.5 when the code layer neuron number reaches 8 ; the $r^{2}$ for all subsections are higher than 0.35 when the code layer neuron number is 1 . For the log response times, the MAE is controlled to be lower than 0.45 in LS, lower than 0.65 in LR and lower than 0.7 in QS; the RMSE is controlled to be lower than 0.6 in LS, lower than 0.9 in LR and lower than 0.95 in QS. In the original response time scale, the MAE is controlled to be lower than 20 in LS, lower than 35 in LR and lower than 45 in QS; the RMSE is controlled to be lower than 35 in LS, lower than 60 in LR and lower than 75 in QS. Note that the $\mathrm{MAE}_{\text {org }}$ and $\mathrm{RMSE}_{\text {org }}$ computed from the empirical data cannot be compared to the ones computed in the simulation study directly. The response time data in the empirical data is recorded in the second unit while the one in the simulation is generated in the minute unit.

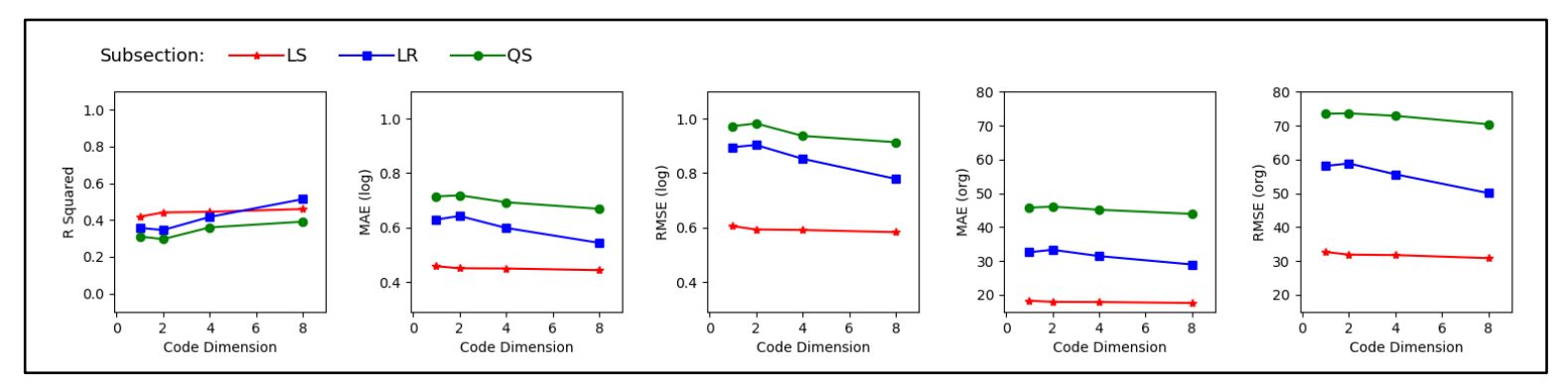

Figure 9 Item Parameter Estimation Performance in Empirical Data

To evaluate the performance of the proposed approach in item parameter estimation, the present study calculated the model fitness for the remained $40 \%$ data using the corresponding models and presented the results in Figure 9. In general, this result is similar to the model fit for training data (see Figure 8), showing an acceptable fitness for the data, hence this approach has an acceptable performance in item parameter estimation.

Since the true speed parameters are unknown, the index $\left|r_{s, \zeta}\right|$ used in the experiment is not applicable here. Instead, the present study plots the estimated speed to show the distribution of them (see Figure 10). Figure 10 shows the speed parameter distribution for the response time model with an 8-dimension code (all the speed parameters were plotted together). As expected, the distribution is close to the response time distribution. 


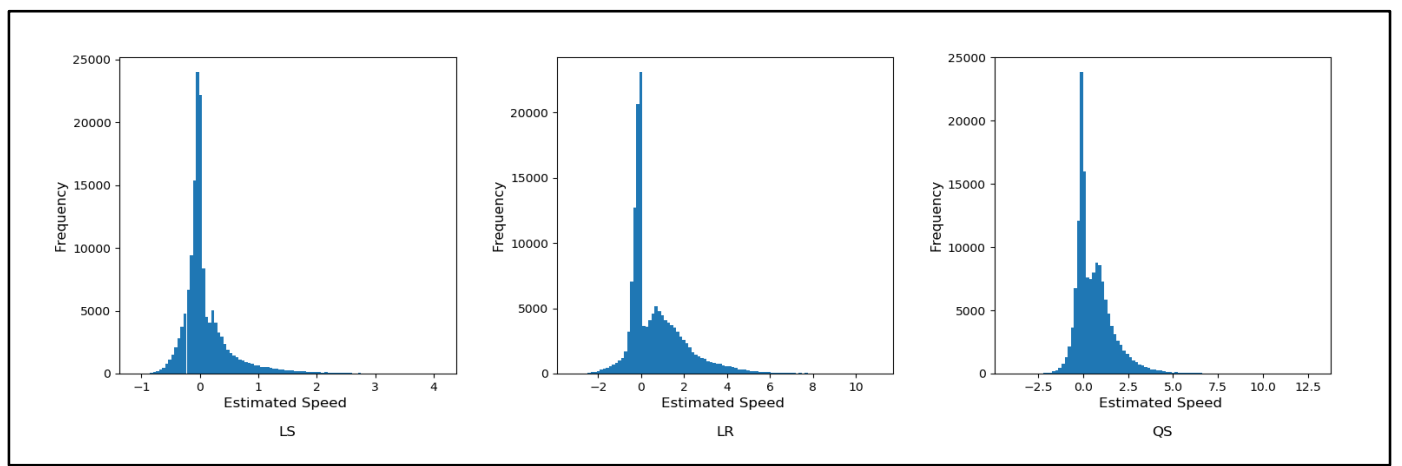

Figure 10 Estimated Speed Distribution in Empirical Data

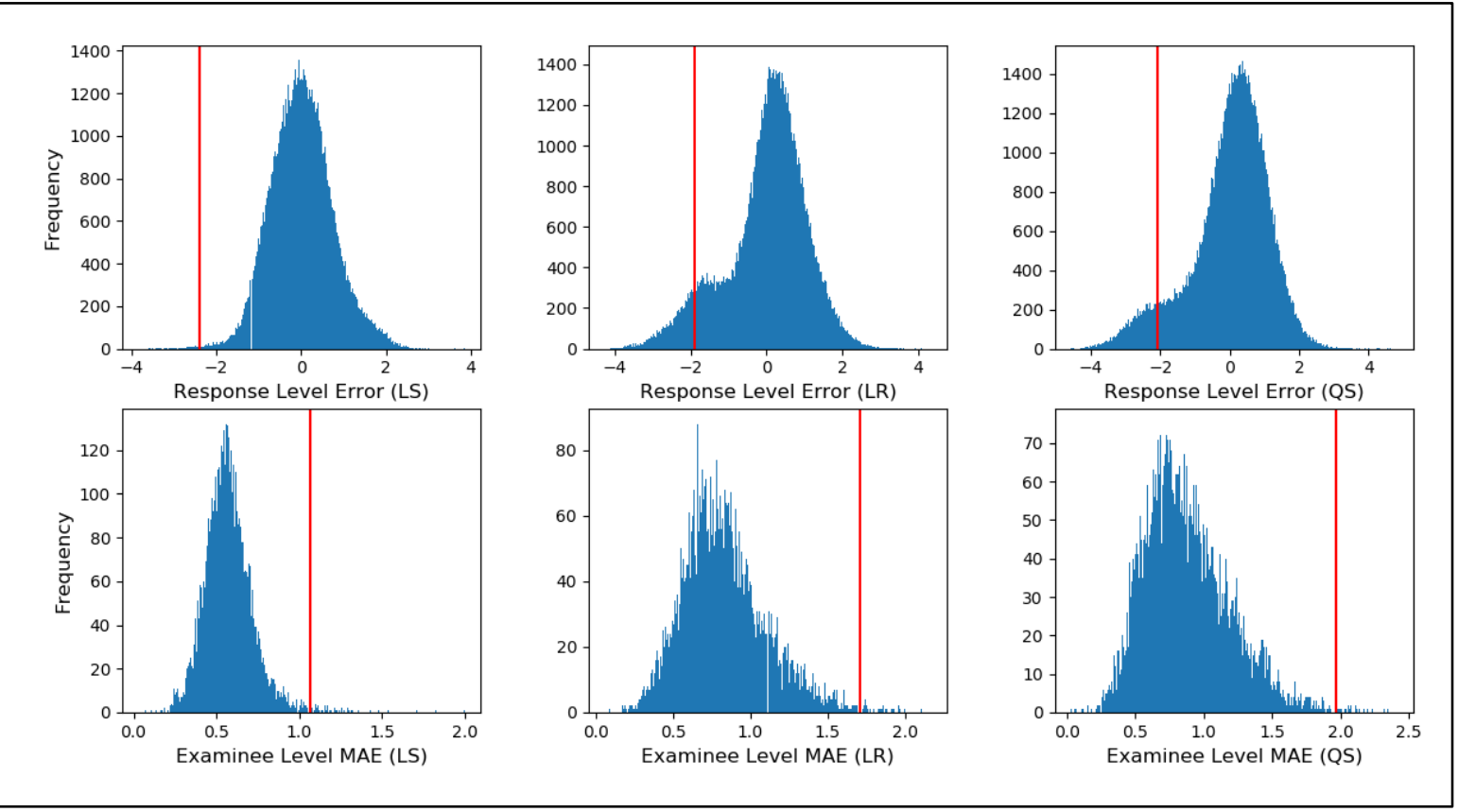

Figure 11 Error Distribution in Empirical Data

The proposed model was also applied to detect anomaly in the empirical data. Since the truly aberrant items and examinees are unknown, the error rates used in the experiment are not applicable here. Instead, the present study plots the error distribution for responses and the mean absolute error distribution for examinees (see Figure 11). In general, the error distributions, particularly the distributions of examinee-level rooted mean squared error, are approximately normally distributed, and the flagged examinees or responses are far away from the distribution centers.

\section{Conclusion}

The present study proposed an autoencoder-based response time model and introduced the application of this model in response time modeling and anomaly detection. The results indicate that this model has an acceptable performance in response time modeling, showing a 
relatively high model fit under most situations. In addition, the proposed approach performs well in item parameter estimation, often getting fitness in the testing data similar to the one in training data. The performance in examinee parameter estimation is good, the estimated speeds have a high correlation strength with the speed parameters used for simulation. For anomaly detection, the proposed approach has an acceptable performance: for aberrant examinee detection, this approach controls the false negative rates at a moderate level and the false positive rates at a low level; for aberrant item detection, this approach controls the false positive rates at a low level, the false negative rates at a moderate level provided the proportion of aberrant items is not high (e.g., 0.6), however, when the aberrant item proportion is high, the false negative rates become very high.

The model fitting performance in the empirical data is not as good as the one in the simulation. This might be explained by the testing type. Since LOFT is a compromise between traditional linear testing and CAT, and different forms are administered to examinees nonadaptively, responses of several forms are completely missing for each examinee. This amount and type of missing may bring great difficulty in modeling.

There are several limitations in this research. First, the proposed response time model is only designed for correctly answered responses, future research could extend the model to be a system which is able to process both correctly and incorrectly answered responses. Second, as the item parameters are the weights in autoencoder, the number of parameters is much more than the one in traditional psychometrician response time models, in other words, the model is very complex. Although complexity is one of nature neural networks have, future research could explore the strategy for designing optimal neural network structure for modeling response times, or develop a feature engineering process in advance to simplify the network. Third, the estimated examinee parameters are not comparable across different tests since the values in the code layer are influenced by the scale and signs of the weights, future research could explore the techniques like weight normalization or batch normalization to make the estimated speed comparable. Fourth, the present study only conducts simulations for CAT, future research could explore the application of the proposed response time model on the linear tests.

In addition, this approach used a simple strategy to deal with the missing in the response time data, replacing with zero or the examinee-level average response times, future research could explore more delicate techniques which can give a better estimation of the missing data. Moreover, although the approach for anomaly detection does not produce low false negative rates, it controls the false positive rates at a low level across conditions, future research could 
explore the possibility of hybridizing this approach with other existing methods to obtain a better detection performance. 


\section{Reference}

Chang, H. H., Qian, J., \& Ying, Z. (2001). a-Stratified multistage computerized adaptive testing with b blocking. Applied Psychological Measurement, 25(4), 333-341.

Cho, K., Van Merriënboer, B., Gulcehre, C., Bahdanau, D., Bougares, F., Schwenk, H., \& Bengio, Y. (2014). Learning phrase representations using RNN encoder-decoder for statistical machine translation. arXiv preprint arXiv:1406.1078.

Choe, E. M., Zhang, J., \& Chang, H. H. (2018). Sequential detection of compromised items using response times in computerized adaptive testing. Psychometrika, 83(3), 650-673.

De Boeck, P., \& Jeon, M. (2019). An overview of models for response times and processes in cognitive tests. Frontiers in psychology, 10, 102.

Eckerly, C. (2017). Detecting preknowledge and item compromise. In G. J. Cizek \& J. A. Wollack (Eds.) Handbook of quantitative methods for detecting cheating on tests (pp. 101-123). New York, NY: Routledge.

Hinton, G., Deng, L., Yu, D., Dahl, G. E., Mohamed, A. R., Jaitly, N., ... \& Kingsbury, B. (2012). Deep neural networks for acoustic modeling in speech recognition: The shared views of four research groups. IEEE Signal processing magazine, 29(6), 82-97.

Lu, L., Shin, Y., Su, Y., \& Karniadakis, G. E. (2019). Dying relu and initialization: Theory and numerical examples. arXiv preprint arXiv:1903.06733.

Najafabadi, M. M., Villanustre, F., Khoshgoftaar, T. M., Seliya, N., Wald, R., \& Muharemagic, E. (2015). Deep learning applications and challenges in big data analytics. Journal of big data, 2(1), 1-21.

Redmon, J., Divvala, S., Girshick, R., \& Farhadi, A. (2016). You only look once: Unified, realtime object detection. In Proceedings of the IEEE conference on computer vision and pattern recognition (pp. 779-788).

Santurkar, S., Tsipras, D., Ilyas, A., \& Mądry, A. (2018, December). How does batch normalization help optimization?. In Proceedings of the 32nd international conference on neural information processing systems (pp. 2488-2498).

Sinharay, S. (2017). Detection of item preknowledge using likelihood ratio test and score test. Journal of Educational and Behavioral Statistics, 42(1), 46-68.

van der Linden, W. J. (2007). A hierarchical framework for modeling speed and accuracy on test items. Psychometrika, 72(3), 287-308. 